\title{
Video Article \\ A Protocol for Detecting and Scavenging Gas-phase Free Radicals in Mainstream Cigarette Smoke
}

\author{
Long-Xi Yu ${ }^{1}$, Boris G. Dzikovski ${ }^{2}$, Jack H. Freed ${ }^{2}$ \\ ${ }^{1}$ CDCF-AOX Lab \\ ${ }^{2}$ National Biomedical Center for Advanced ESR Technology (ACERT), Department of Chemistry and Chemical Biology, Cornell University
}

Correspondence to: Jack H. Freed at jhf3@cornell.edu

URL: https://www.jove.com/video/3406

DOI: doi:10.3791/3406

Keywords: Bioengineering, Issue 59, Cigarette smoke, free radical, spin-trap, ESR

Date Published: 1/2/2012

Citation: Yu, L.X., Dzikovski, B.G., Freed, J.H. A Protocol for Detecting and Scavenging Gas-phase Free Radicals in Mainstream Cigarette Smoke. J. Vis. Exp. (59), e3406, doi:10.3791/3406 (2012).

\section{Abstract}

Cigarette smoking is associated with human cancers. It has been reported that most of the lung cancer deaths are caused by cigarette smoking ${ }_{5,6,7,12}$. Although tobacco tars and related products in the particle phase of cigarette smoke are major causes of carcinogenic and mutagenic related diseases, cigarette smoke contains significant amounts of free radicals that are also considered as an important group of carcinogens $s^{9,10}$. Free radicals attack cell constituents by damaging protein structure, lipids and DNA sequences and increase the risks of developing various types of cancers. Inhaled radicals produce adducts that contribute to many of the negative health effects of tobacco smoke in the lung ${ }^{3}$. Studies have been conducted to reduce free radicals in cigarette smoke to decrease risks of the smoking-induced damage. It has been reported that haemoglobin and heme-containing compounds could partially scavenge nitric oxide, reactive oxidants and carcinogenic volatile nitrosocompounds of cigarette smoke ${ }^{4}$. A 'bio-filter' consisted of haemoglobin and activated carbon was used to scavenge the free radicals and to remove up to $90 \%$ of the free radicals from cigarette smoke ${ }^{14}$. However, due to the cost-ineffectiveness, it has not been successfully commercialized. Another study showed good scavenging efficiency of shikonin, a component of Chinese herbal medicine ${ }^{8}$. In the present study, we report a protocol for introducing common natural antioxidant extracts into the cigarette filter for scavenging gas phase free radicals in cigarette smoke and measurement of the scavenge effect on gas phase free radicals in mainstream cigarette smoke (MCS) using spin-trapping Electron Spin Resonance (ESR) Spectroscopy ${ }^{1,2,14}$. We showed high scavenging capacity of lycopene and grape seed extract which could point to their future application in cigarette filters. An important advantage of these prospective scavengers is that they can be obtained in large quantities from byproducts of tomato or wine industry respectively ${ }^{11,13}$

\section{Video Link}

The video component of this article can be found at https://www.jove.com/video/3406/

Protocol

\section{Materials}

All solvents used in this work were reagent grade. The spin trap, including N-tert-butyl-a-phenylnitrone (PBN) and the standard spin label 2,2,6,6tetramethyl-1-piperinyoxyl (TEMPO) were obtained from Sigma and were used as supplied. The plant antioxidants were obtained commercially from Swanson, Inc. USA.

\section{Preparation of cigarette smoke and analysis of free radicals}

1. To introduce antioxidants into the filter, the antioxidants pycnogenol and grape seed extract were first dissolved in $95 \%$ ethanol, while lycopene was dissolved in acetone. The solvent volumes were different depending on the antioxidant solubility.

2. The amount of antioxidants of $0.4 \mathrm{mg} /$ filter was used. They were then coated with $10 \mathrm{mg}$ of activated carbon. For this purpose activated carbon was stirred for $\sim 12$ hrs with the antioxidant solution in anaerobic conditions, filtered and dried under vacuum.

3. Antioxidants were then introduced into the conventional acetate filter (CA filter). For this purpose, the filter was cut into two pieces. The coated plant antioxidants were inserted between two pieces of the filter and wrapped with a piece of tape to form a filter - antioxidant -filter sandwich (Fig. 1A). This combined filter was subsequently attached to cigarette rods containing tobacco. The control filter was made in the same way as the antioxidant filter except no antioxidant was added.

4. Prior to the smoking simulation, the research cigarettes were unpacked and kept in a constant humidity environment $\left(20{ }^{\circ} \mathrm{C}, 60 \%\right.$ relative humidity) using a saturated $\mathrm{NaBr}$ solution for a minimum of 2 days.

5. Smoking simulation for routine analysis was performed at ambient temperature using a single-port smoking device which, as shown in Fig.1, consists of a water aspirator or GAST DOA-P104-BN Vacuum Pump/Air Compressor (Benton Harbor, Mich) connected to the spin-trapping 
assembly through a T-junction with one end open. Puffs were performed by plugging the open end which remained open between puffs. The gas flow was checked by flow meter and set at $\sim 2.2 \mathrm{SCFH}=17.5 \mathrm{~mL} / \mathrm{s}$ by adjusting a valve placed between the pump and the vent.

6. The research cigarettes were smoked under the condition of puff volume of $35 \mathrm{~mL}$ for a duration of 2 seconds repeated every 60 seconds, similar to ${ }^{1}$. Smoking simulation for quantitative estimation of gas-phase free radicals was performed in conjunction with the spin trapping system as shown in Fig. 1.

7. Ten intense puffs ( $35 \mathrm{~mL} /$ puff) were taken for each cigarette. Gas-phase free radicals were collected by passing the MCS through a Cambridge filter pad and then introduced into the spin trapping solution (0.05 M PBN in benzene, $2.0 \mathrm{~mL})$.

8. After the last puff, the bubbled trapping solution was readjusted to its initial volume $(2.0 \mathrm{~mL})$ with the same benzene. An aliquot was transferred into a $\sim 250 \mathrm{~mm}$ long, $3 \mathrm{~mm}$ ID glass tube sealed at one end.

9. The trapping solution was deoxygenated using a freeze-pump-thaw procedure. It was frozen under liquid nitrogen, and a vacuum was applied. Then it was thawed under argon atmosphere, allowing trapped gas bubbles to escape, and frozen again. After this cycle was repeated three times, the tube was flame-sealed under vacuum and used in further ESR measurements. This step is required because oxygen, which is well soluble in benzene, broadens ESR lines of organic radicals. Deoxygenation dramatically improves the signal-to-noise ratio.

10. X-Band ESR spectra were recorded on a Bruker EMX spectrometer at a frequency of $9.34 \mathrm{GHz}$ under standard conditions. The spectrometer settings used in most experiments were: center field 3312.5G, scan width $80 \mathrm{G}$, modulation amplitude $0.5 \mathrm{G}$, time constant 82 microseconds, scan time $40 \mathrm{sec}$.

11. The spin trap adducts are relatively stable at the experiment conditions, which in some cases required 25 accumulations, which typically took $\sim 20 \mathrm{~min}$. However, after $12 \mathrm{hrs}$ the intensity of the ESR signals in benzene solution decreased by a factor of $\sim 5$.

12. To quantify the concentration of the trapping adduct, its initial first-derivative ESR spectrum was integrated. The resulting absorption spectrum shows a broad singlet background, most likely due to soot/tar products contained in the smoke.

After subtraction of this background the separated triplet of the trapping adduct was integrated one more time (Fig. 2).

\section{Representative Results}

Most of the free radicals in burning cigarette-produced smoke (gas phase) are instantaneous and unstable. In order to observe these radicals a spin trap technique is employed. It captures gas-phase free radicals by transforming them into a spin adduct which is more stable and can be detected by ESR (Fig.1). In the present study, the spin-trap solution of $0.05 \mathrm{M}$ PBN was used to collect smoke gas phase free radicals, which are a mixture of oxygen- and carbon- centered radicals which are difficult to separate ${ }^{15}$. In our case, however, the observed hyperfine splitting constants $a_{N}=13.7 \mathrm{G}$ and $a_{H}=1.95 \mathrm{G}$ are very similar to the corresponding values for trapping adducts of alkoxyl free radicals (RO.) $)^{2}$, suggesting that they are the major product. We showed that weak ESR signals and low reproducibility observed in our initial measurements (Fig. 2) were due to the moisture in the smoke flow. To resolve this problem, we added a liquid nitrogen (LN2) trap between the Cambridge filter and the spin trap solution. The $\mathrm{LN}_{2}$ trap removed water from the MCS flow by quick freezing and capturing it on the inner wall of the glass tube. This greatly improved ESR signals and allowed for highly reproducible results (Fig. 3).

The amount of trapped free radicals was determined using a reference sample. For control samples without antioxidants, the typical adduct concentration in benzene estimated by comparison of its double integral spectrum with a double integral spectrum for known concentration of TEMPO was $1.24 \mu \mathrm{M}$ (Fig.2). Since the amount of air passed through each cigarette during smoking was $\sim 350 \mathrm{ml}$, this gives an estimate for the free radical concentration in the gas phase of MCS of $\sim 7.1 \times 10^{-9} \mathrm{M}$, and a total number of radicals trapped from the gas phase of a whole cigarette of $\sim 1.5 \times 10^{15}$. An estimate for the total amount of free radicals in the smoke of a whole cigarette, including both gas and particulate phase, was $\sim 10^{16}$ free radicals ${ }^{9}$.

Different levels of the scavenging effect of plant antioxidants on gas-phase free radicals in main stream cigarette smoke were observed. Their scavenging rates were presented in Fig. 4. Lycopene and grape seed extract showed the highest rates while lower rate was observed for pycnogenol (Fig.4).

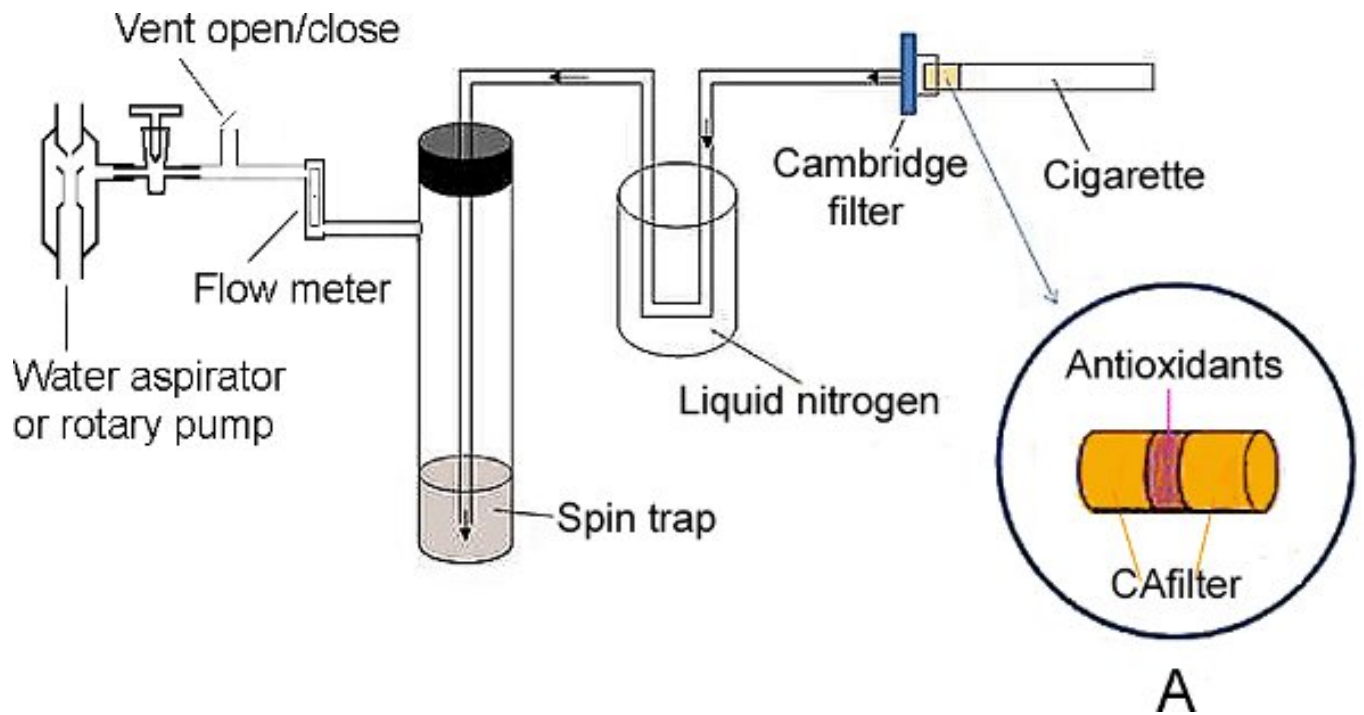

Figure 1. A diagram of improved smoking simulation design for collecting gas-phase free radicals in mainstream cigarette smoke (MCS) using spin trap. MCS was drawn by a water aspirator through CA filter, then Cambridge filter (yellow filter) and passed through a liquid nitrogen trap to 
remove $\mathrm{H}_{2} \mathrm{O}$. The gas-phase products finally went to spin trap and bubbled through the spin trap solution. Plant antioxidant was placed between two pieces of conventional acetate filters (Enlarged in circle A) attached to cigarette to scavenge free radicals in MCS.
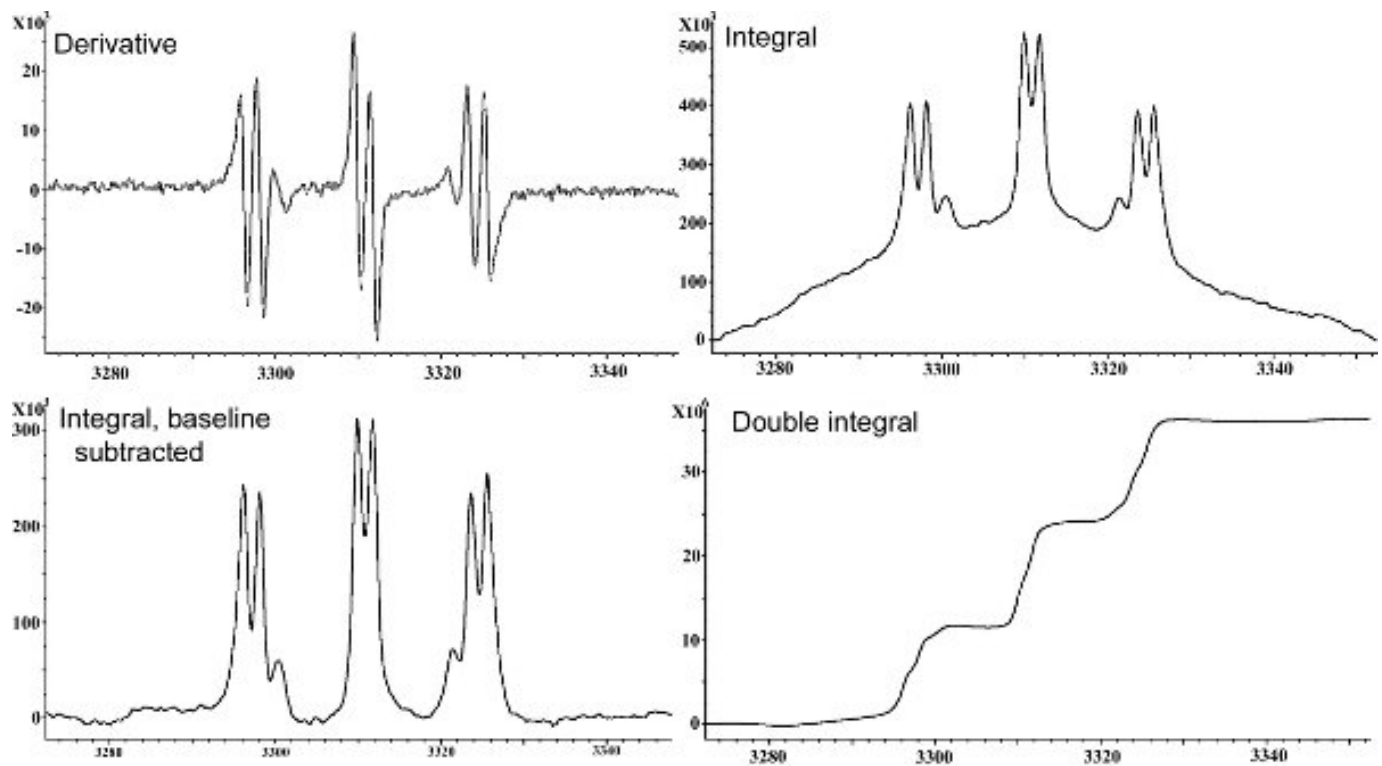

Figure 2. A quantitative estimate for the spin-trapping adduct concentration in benzene requires subtracting a broad background signal from the first integral spectrum. The hyperfine splitting parameters for the spin-trapping adduct are $a_{H}=1.95 \mathrm{G}, a_{N}=13.7 G$.

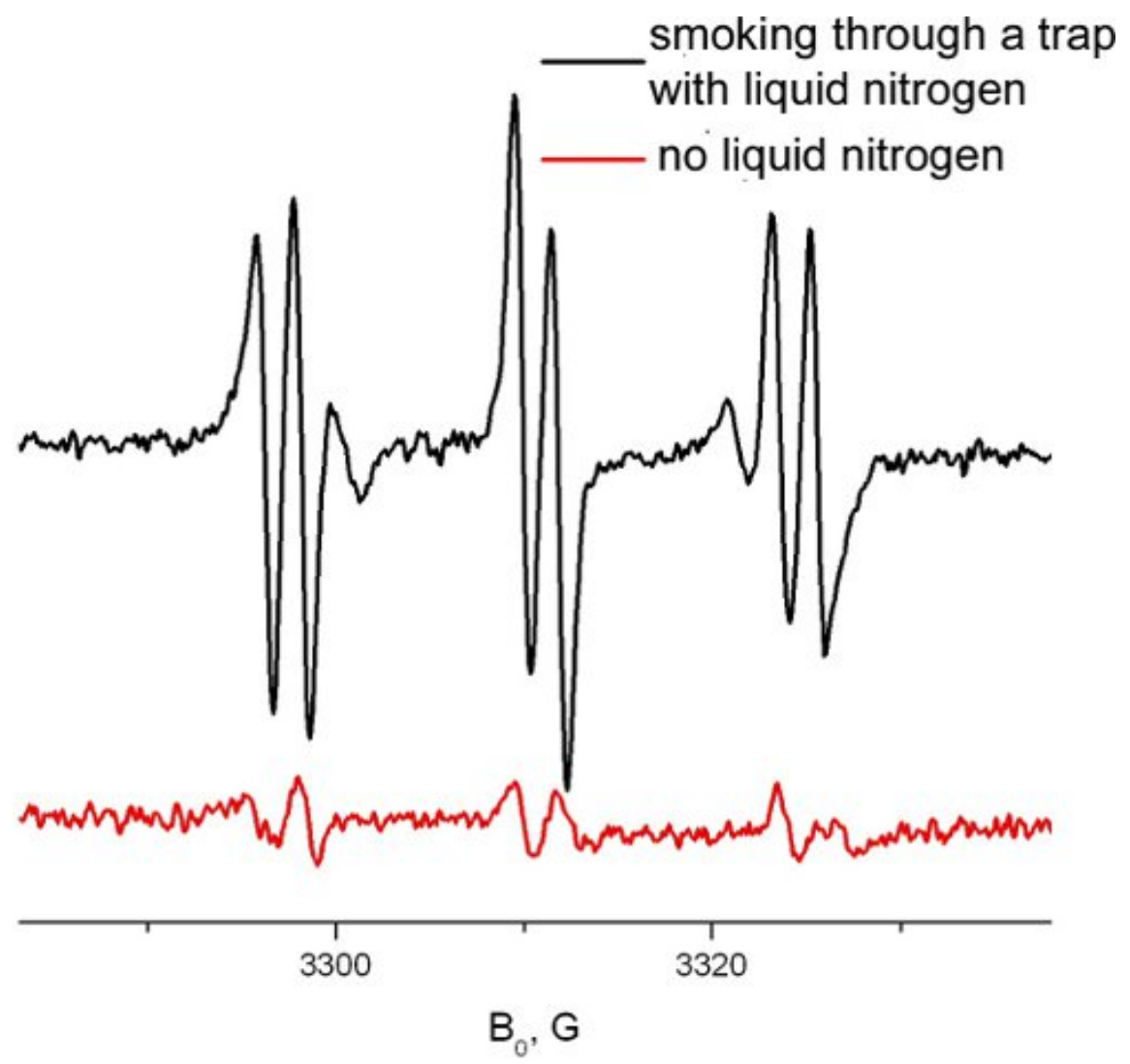

Figure 3. Passing through LN2 trap substantially improves the quality of ESR signals obtained by spin-trapping of MCS. 


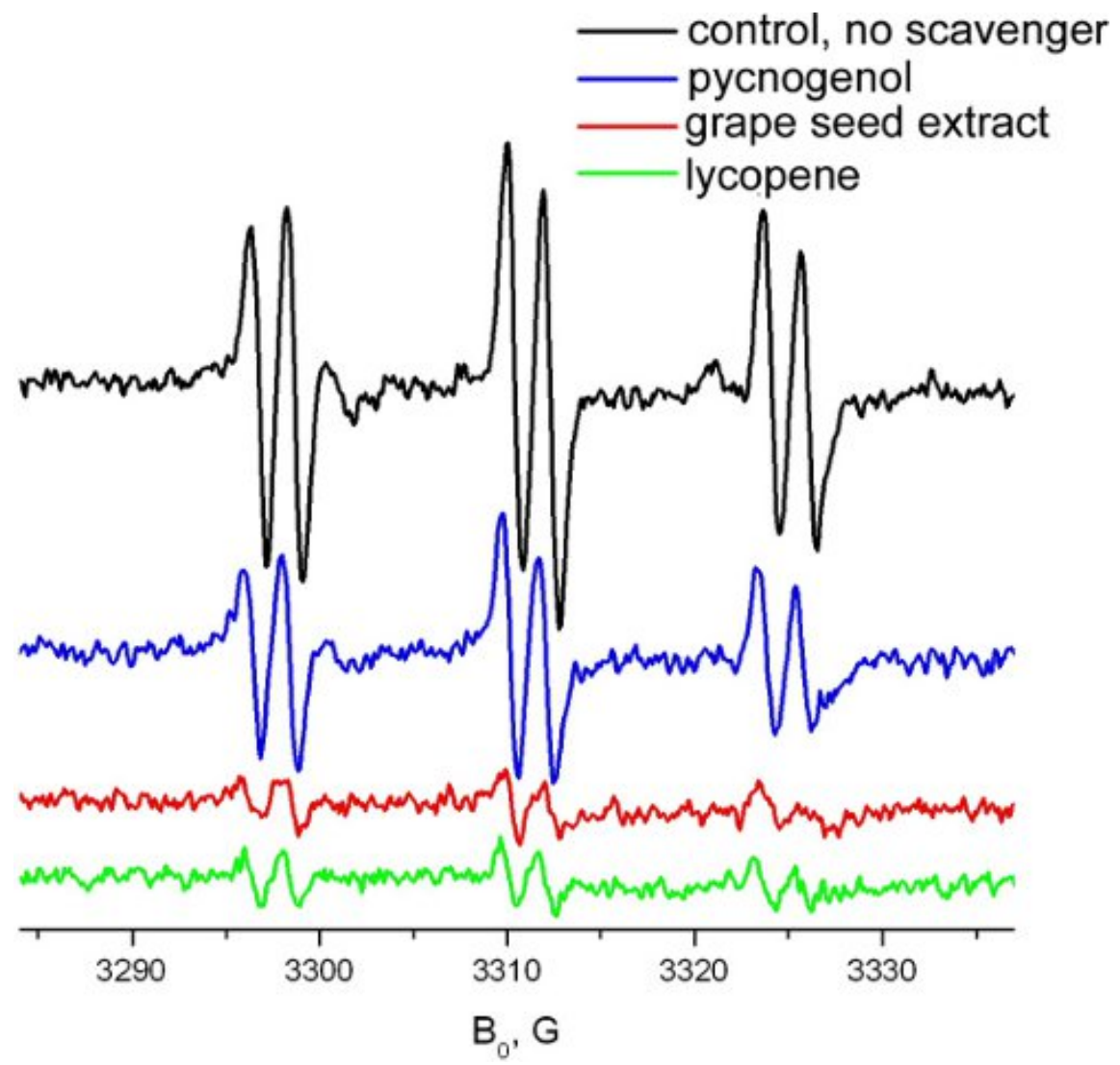

Figure 4. Effect of natural antioxidants on the concentration of free radicals in MCS. The relative signal intensities are: control - $100 \%$, pycnogenol $-55 \%$, grape seed extract - $12 \%$, lycopene - $10 \%$.

\section{Discussion}

A reliable estimate of the effect of different free-radical scavengers in tobacco smoke requires a reproducible technique for quantitative detection of free radicals. Previously ${ }^{1}$, it has been shown that small volumes of higher concentration spin trap solutions in non-polar solvents are most effective at trapping free radicals from tobacco smoke. Cigarette smoke always contains water vapor from combustion of organic compounds and the residual moisture in tobacco which can end up in the trapping solvent. This admixture of water in the PBN spin trap solution substantially decreases the lifetime of the spin trapping adducts and the intensity of their ESR signals. Removing this moisture by simple passing MCS through a U-shaped tube cooled with liquid nitrogen dramatically improved the quality of ESR spectra in our experiments, even though some fraction of gas-phase radicals might be also trapped on the frozen surface.

Using this technique we compared relative efficiency of different radical-scavenging natural compounds introduced into cigarette filters. We found that lycopene and grape seed extract immediately after incorporating into cigarette filters are able to scavenge up to $90 \%$ of free radicals from the gas phase MSC. Such high scavenging capacity ranks these cheap readily available natural compounds among the most efficient reported free radical protectors like haemoglobin and shikonine ${ }^{8,14}$. Yet, in our experiments cigarette filters loaded with studied natural antioxidants lost a noticeable part of their scavenging capacity after a week of storage at room temperature. Solving this problem can stimulate future application of lycopene and grape seed extract in commercial cigarette filters.

\section{Disclosures}

No conflicts of interest declared.

\section{Acknowledgments}

This work was supported by the National Institute of Health, grant No. NIH/NCRR P41-RR 016292 (for ACERT).

\section{References}

1. Baum, S.L., Anderson, I.G.M., Baker, R.R, Murphy, D.M., \& C.C., Rowlands. Electron spin resonance and spin trap investigation of free radicals in cigarette smoke: development of a quantification procedure. Analytica Chimica. Acta. 481, 1-13 (2003).

2. Bluhm, A.L., Weinstein, J., \& Sousa, J.A. Free radicals in tobacco smoke. Nature. 229, 500 (1971). 
3. Church, D.F. \& Pryor W.A. Free-radical chemistry of cigarette smoke and its toxicological implications. Environ. Health. Perspect. 64, 111-126 (1985).

4. Deliconstantinos, G., Villiotou, V., \& Stavrides, J.C. Scavenging effects of hemoglobin and related heme containing compounds on nitric oxide, reactive oxidants and carcinogenic volatile nitrosocompounds of cigarette smoke. a new method for protection against the dangerous cigarette constituents. Anticancer. Res. 14, $2717-2726$ (1994).

5. Hecht, S.S. Tobacco smoke carcinogens and lung cancer. Journal of the National Cancer Institute. 91, 1194-1210 (1999).

6. Kodama, M., Kaneko, M., Aida, M., Inoue, F., Nakayama, T., \& Akimoto, H. Free radical chemistry of cigarette smoke and its implication in human cancer. Anticancer. Res. 17, 433-437 (1997).

7. Nair, A.K. \& Brandt, E.N. Effects of smoking on health care costs. Journal of Oklahoma State Medical Association. 93, 245-250 (2000).

8. Nishizawa, M., Kohno, M., Nishimura, M., Kitagawa, A., \& Niwano, Y. Presence of Peroxyradicals in Cigarette Smoke and the Scavenging Effect of Shikonin, a Naphthoquinone Pigment. Chem. Pharm. Bull. 53, 796-799 (2005).

9. Pryor, W.A. Cigarette smoke and the involvement of free radical reactions in chemical carcinogenesis. Br. J. Cancer. 8, 19-23 (1987).

10. Pryor, W.A. Cigarette smoke radicals and the role of free radicals in chemical carcinogenicity. Environ. Health. Perspect. 105, 875-882 (1997).

11. Rozzi, N.I., Singh, R.K., Vierling, R.A., \& Watkins, B.A. Supercritical fluid extraction of lycopene from tomato processing byproducts. J. Agric. Food. Chem. 50, 2638-2643 (2002).

12. Shopland, D.R. Tobacco use and its contribution to early cancer mortality with a special emphesis on cigarette smoking. Environmental. Health. Perspectives. 103, 131-142 (1995).

13. Shrikhandle, A.J. Wine products with health benefits. Food Res. Int. 33, 469-474 (2000).

14. Valavanidis, A. \& Haralambous, E.A comparative study by electron paramagnetic resonance of free radical species in the mainstream and sidestream smoke of cigarettes with conventional acetate filters and 'bio-filters'. Redox. Rep. 6, 161-171 (2001).

15. Valavanidis, A., Vlachogianni, T., \& Fiotakis, K. Tobacco smoke: involvement of reactive oxygen species and stable free radicals in mechanisms of oxidative damage, carcinogenesis and synergistic effects with other respirable particles. International Journal of Environmental Research and Public Health. 6, 445-462 (2009). 